\title{
RESEARCH
}

Open Access

\section{Impact of a clinical decision support tool on prediction of progression in early-stage dementia: a prospective validation study}

Marie Bruun ${ }^{1 *}\left(\mathbb{D}\right.$, Kristian S. Frederiksen ${ }^{1}$, Hanneke F. M. Rhodius-Meester ${ }^{2}$, Marta Baroni ${ }^{3}$, Le Gjerum', Juha Koikkalainen ${ }^{4}$, Timo Urhemaa ${ }^{5}$, Antti Tolonen ${ }^{5}$, Mark van Gils ${ }^{5}$, Daniel Rueckert ${ }^{6}$, Nadia Dyremose ${ }^{1}$, Birgitte B. Andersen ${ }^{1}$, Afina W. Lemstra ${ }^{2}$, Merja Hallikainen ${ }^{7,8}$, Sudhir Kurl ${ }^{7,8}$, Sanna-Kaisa Herukka ${ }^{7,8}$, Anne M. Remes ${ }^{9,10}$, Gunhild Waldemarr ${ }^{1}$, Hilkka Soininen ${ }^{7,8}$, Patrizia Mecocci ${ }^{3}$, Wiesje M. van der Flier ${ }^{2}$, Jyrki Lötjönen ${ }^{4}$ and Steen G. Hasselbalch ${ }^{1}$

\begin{abstract}
Background: In clinical practice, it is often difficult to predict which patients with cognitive complaints or impairment will progress or remain stable. We assessed the impact of using a clinical decision support system, the PredictND tool, to predict progression in patients with subjective cognitive decline (SCD) and mild cognitive impairment (MCl) in memory clinics.

Methods: In this prospective multicenter study, we included 429 patients with SCD $(n=230)$ and $\mathrm{MCl}(n=199)$ (female 54\%, age $67 \pm 9$, MMSE $28 \pm 2$ ) and followed them for at least 12 months. Based on all available patient baseline data (demographics, cognitive tests, cerebrospinal fluid biomarkers, and MRI), the PredictND tool provides a comprehensive overview of the data and a classification defining the likelihood of progression. At baseline, a clinician defined an expected follow-up diagnosis and estimated the level of confidence in their prediction using a visual analogue scale (VAS, 0-100\%), first without and subsequently with the PredictND tool. As outcome measure, we defined clinical progression as progression from SCD to $\mathrm{MCl}$ or dementia, and from $\mathrm{MCl}$ to dementia. Correspondence between the expected and the actual clinical progression at follow-up defined the prognostic accuracy.
\end{abstract}

Results: After a mean follow-up time of $1.7 \pm 0.4$ years, 21 (9\%) SCD and 63 (32\%) MCl had progressed. When using the PredictND tool, the overall prognostic accuracy was unaffected $(0.4 \%, 95 \% \mathrm{Cl}-3.0 \% ;+3.9 \% ; p=0.79)$. However, restricting the analysis to patients with more certain classifications $(n=203)$, we found an increase of $3 \%$ in the accuracy $(95 \% \mathrm{Cl}-0.6 \% ;+6.5 \% ; p=0.11)$. Furthermore, for this subgroup, the tool alone showed a statistically significant increase in the prognostic accuracy compared to the evaluation without tool $(6.4 \%, 95 \% \mathrm{Cl} 2.1 \%$; $10.7 \%$; $p=0.004)$. Specifically, the negative predictive value was high. Moreover, confidence in the prediction increased significantly $(\triangle \mathrm{VAS}=4 \%, p<.0001)$.

Conclusions: Adding the PredictND tool to the clinical evaluation increased clinicians' confidence. Furthermore, the results indicate that the tool has the potential to improve prediction of progression for patients with more certain classifications.

Keywords: Dementia, Alzheimer's disease, Conversion, Progression, Mild cognitive impairment, Subjective cognitive decline, CDSS, Computer-assisted

\footnotetext{
* Correspondence: marie.bruun@regionh.dk

${ }^{1}$ Danish Dementia Research Centre, Neuroscience Centre, Department of

Neurology, Rigshospitalet, University of Copenhagen, Copenhagen University

Hospital, Blegdamsvej 9, 2100 Copenhagen, Denmark

Full list of author information is available at the end of the article
}

(c) The Author(s). 2019 Open Access This article is distributed under the terms of the Creative Commons Attribution 4.0 International License (http://creativecommons.org/licenses/by/4.0/), which permits unrestricted use, distribution, and

reproduction in any medium, provided you give appropriate credit to the original author(s) and the source, provide a link to the Creative Commons license, and indicate if changes were made. The Creative Commons Public Domain Dedication waiver (http://creativecommons.org/publicdomain/zero/1.0/) applies to the data made available in this article, unless otherwise stated. 


\section{Background}

A large proportion of patients referred to memory clinics present with mild cognitive impairment (MCI) or subjective cognitive decline (SCD). Patients with SCD show no detectable deficit in cognition, but have an increased risk of progression and of developing Alzheimer's disease (AD) dementia [1-3], whereas the term MCI refers to patients already showing objective signs of cognitive dysfunction [4]. The estimated annual conversion rate for patients with MCI is 5-10\% [5]. However, not all MCI patients will progress and studies have shown that over a period of 10 years, less than $50 \%$ will have developed dementia $[4,5]$. For clinicians, it is often difficult to identify which patients will remain stable or progress [2, 5]. However, individualized risk management and early detection of individuals with a progressive condition are important for providing optimal counseling, care, and treatment $[6,7]$.

Pathophysiological abnormalities are known to precede clinical symptoms of $\operatorname{AD}[3,8]$. Positive diagnostic tests, such as decreased amyloid- $\beta$ 1-42 (A $\beta 42)$ and elevated tau proteins in cerebrospinal fluid (CSF), APOE genotype, and atrophy on magnetic resonance imaging (MRI), are associated with an increased risk of progression in patients with MCI and SCD [2, 3, 9-13]. Nevertheless, clinicians struggle to translate this information into clinical decision-making and accurately predict whether the individual patient will progress or not. The latest diagnostic criteria acknowledge the role of biomarkers in predementia stages of AD [4]. However, guidance regarding the prognostic value of the biomarkers and how to combine them optimally is still inadequate $[10,14]$. Therefore, clinicians may experience ethical dilemmas when applying biomarkers, as well as communicating biomarker results, without knowing the actual prognostic consequences for the individual patient [15]. Modern clinical decision support systems provide a potential solution as they can assess probabilities of individuals rather than provide only statistical differences at a group level. Furthermore, the decision support systems can assist clinicians in clinical practice by providing an objective and consistent comparison of multivariate and multimodal data [16].

Most classifiers use mainly MRI or combined MRI and CSF biomarkers [17], but classifiers including more clinical variables into the progression model have also been introduced $[18,19]$. The disease state index (DSI) classifier integrates all available baseline data (demographics, cognitive tests, CSF biomarkers, and MRI visual and computed ratings) and provides an index defining the likelihood of progression for the individual patient [19]. Previously, using retrospective data, we have shown that this classifier could discriminate between stable and progressive conditions for $\mathrm{MCI}$, as well as SCD patients
[20-25]. In this study, we used a decision support tool, the PredictND tool, which apart from the likelihood index of the DSI classifier, also gives the clinician an additional comprehensive visual overview and easily interpretable analysis of all data $[19,26]$. In general, decision support tools predicting progression of MCI have predominately been tested on retrospective $\mathrm{AD}$ cohorts $[17,27,28]$. However, to establish which impact decision support tools may have on the complex decision-making in daily clinical practice, evaluation in clinical settings is needed.

In this prospective multicenter study, we assessed the clinical impact of the PredictND tool on the clinician's prediction of clinical progression for patients with SCD and MCI in a mixed memory clinic cohort by comparing the prognostic evaluation without and with the PredictND tool. Our hypothesis was that aid from the PredictND tool would increase the number of correct predictions and the clinicians' confidence in the predictions.

\section{Methods \\ Study design and participants}

We recruited patients from four European memory clinics as part of the PredictND project described in detail elsewhere [29]. The patients were enrolled consecutively from March 2015 to June 2016 and followed for a minimum of 1 year. The included patients were diagnosed with either SCD or MCI, had a baseline Mini-Mental State Examination (MMSE) $\geq 18$, Clinical Dementia Rating $(\mathrm{CDR}) \leq 1.0$, and a T1-weighted MRI at or above 1.5 Tesla within the last 6 months with a decent image resolution (slice thickness $<2.5 \mathrm{~mm}$ ). The exclusion criteria were a major psychiatric disorder, excessive alcohol intake, or substance abuse within the last 2 years, and other brain disorders, which could explain the cognitive problems. We recruited 493 patients, of whom 29 dropped out before the follow-up visit and 35 were excluded, leaving a total number of 429 patients with SCD $(n=230)$ and MCI $(n=199)$ available for analysis (Fig. 1).

All patients underwent a standard multidisciplinary diagnostic screening program including medical history, neurological and physical examination, cognitive testing, blood screening, and an MRI scan. When considered clinically relevant, the assessment program was supplemented with additional tests, such as CSF biomarkers, 18F-fluorodeoxyglucose positron emissions tomography (18F-FDG-PET), or amyloid PET. Patients with cognitive complaints were diagnosed with SCD if the criteria for MCI, dementia, or other disorders are known to cause cognitive problems were not met. MCI was diagnosed according to the National Institute on Aging-Alzheimer's Association (NIA-AA) criteria [4], 


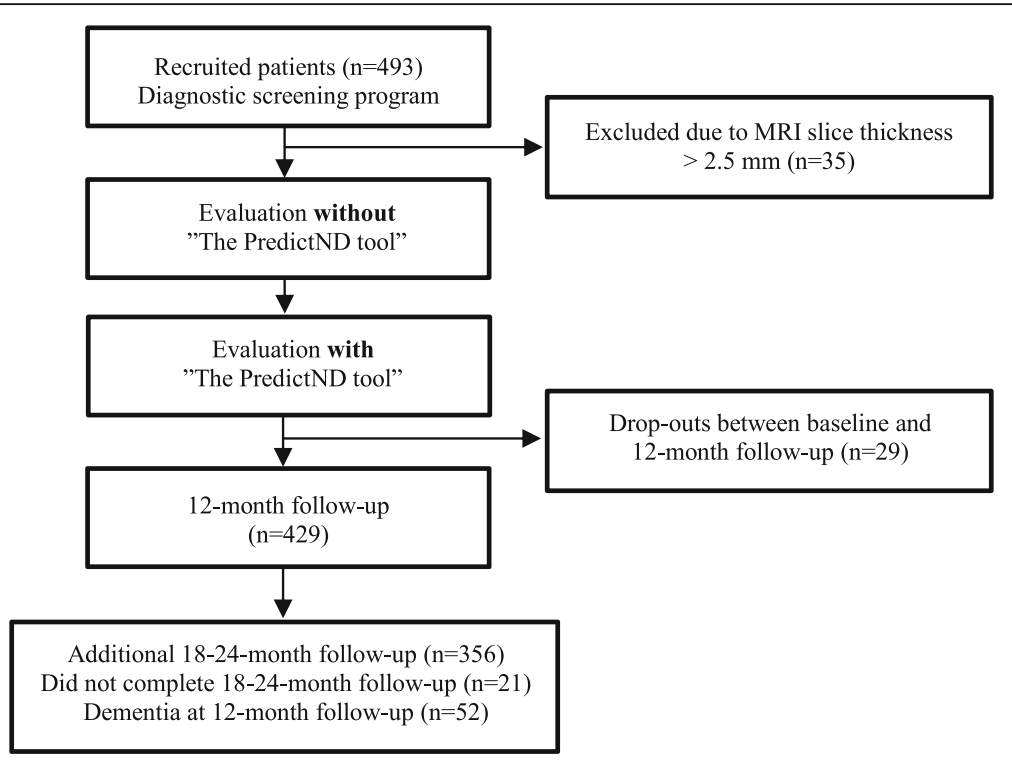

Fig. 1 Flow diagram of the study

whereas $\mathrm{AD}$ dementia was diagnosed according to NIA-AA criteria for AD dementia [30], and other types of dementia were diagnosed using established clinical criteria [31-33].

The study was approved by the local Medical Ethical Committee in all four centers. All patients provided written informed consent for their data to be used for research purposes.

\section{Assessment of impact}

The study was designed to mimic clinical practice. Therefore, a single clinician performed the diagnostic evaluation according to usual practice based on a clinical impression, all available information from medical history, clinical investigations, paraclinical results, and scans. For all patients, the clinician stated the current diagnosis $(\mathrm{SCD} / \mathrm{MCI})$, the expected follow-up diagnosis (SCD/MCI/dementia), and their level of confidence in the estimated prediction as high, moderate, or low, and on a visual analogue scale (VAS) from 0 to $100 \%$. The same (single) clinician re-evaluated the patients using the same available information and test results but also assisted by the PredictND tool. Again, the expected follow-up diagnosis and the clinician's level of confidence in the prediction were recorded. All evaluations were performed as close to baseline as possible. For the clinician to recall the clinical impression of the patient, we aimed to perform the re-evaluations within 30 days from the initial evaluation without tool (median $=28$, IQR 0-123). The evaluations were performed by clinicians $(n=8)$, who had all received basic training on how to use the PredictND tool.
All patients had a clinical follow-up visit after 12 months. If the patient had progressed to dementia, no further follow-up was conducted, whereas patients with SCD or MCI were followed for additional 6-12 months (Fig. 1). At each follow-up visit, including at least MMSE, CDR, and a clinical interview, a follow-up reference diagnosis was determined by a clinician who had no knowledge of the baseline prediction. In a subset of patients $(n=21,5 \%)$, a clinical follow-up visit was not possible, and the diagnostic evaluation was based on a telephone interview. As outcome measure, we defined clinical progression as progression from SCD to MCI or dementia, and from MCI to dementia as diagnosed at follow-up. The expected follow-up diagnosis estimated at baseline compared to the baseline diagnosis defined the clinicians' prediction of progression as either stable or progression. Correspondence between the prediction of progression and the actual clinical progression defined the prognostic accuracy.

\section{The PredictND tool}

The PredictND tool is a clinical decision support tool designed to assist clinicians in differential diagnosis of dementia and to predict whether the condition will progress or remain stable $[19,26,34,35]$. The tool uses a data-driven classifier, which provides a scalar disease state index (DSI) value between zero and one. In this study, DSI indicates the likelihood of progression [19]. The model was developed based on training data from patients with established diagnoses of $\mathrm{AD}$ and controls. A DSI value close to zero indicates that a given patient resembles controls in the database and is more likely to remain stable, whereas a DSI value close to one indicates 
a high likelihood of progression to dementia due to $\mathrm{AD}$. Thus, patients with a low or high DSI value are typically more likely correctly classified than patients with medium DSI values. The DSI classifier is described in detail elsewhere $[19,26]$.

The DSI analysis can handle different types of variables, such as demographic information, cognitive tests, CSF biomarkers, APOE genotyping, and MRI visual and computed ratings, and tolerate missing data [26, 35-37]. The DSI is computed using the following methods: (1) Each variable of the patient's data is compared with the training data using a fitness function defined as $f(x)=$ $\mathrm{FN}(x) /(\mathrm{FN}(x)+\mathrm{FP}(x)$, where $\mathrm{FN}(x)$ is the false negative errors and $\mathrm{FP}(x)$ the false positive errors in the training data, when using $x$ as the classification cutoff value. (2) A relevance function for each variable is defined as relevance $=$ sensitivity + specificity -1 . (3) Finally, the fitness values weighted according to their relevance produce a composite DSI defined through DSI $=\Sigma$ (relevance $\times$ fitness $) / \Sigma$ relevance; see a detailed description in $[19,26]$.

A graphical tree structure, called the disease state fingerprint (DSF), visualizes how each test or biomarker contributes to the DSI classification $[19,26]$. The fit of the patient data to the training data is displayed on a color scale (blue shades indicating controls and red shades $\mathrm{AD}$ ) and the weight by which each data point contributes to the prediction with the sizes of the boxes. This makes interpretation of the result easier for the clinician. Figure 2 shows examples of DSF visualizations from the PredictND tool.

\section{Clinical data}

Cognition was assessed using a standardized cognitive test battery, as presented in Table 2 and described in [29]. Lumbar puncture was performed on clinical

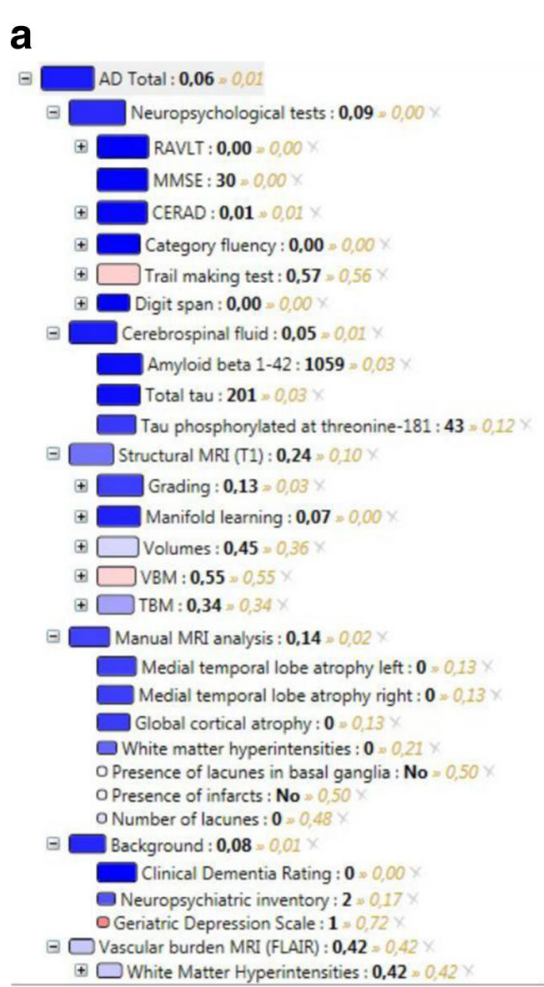

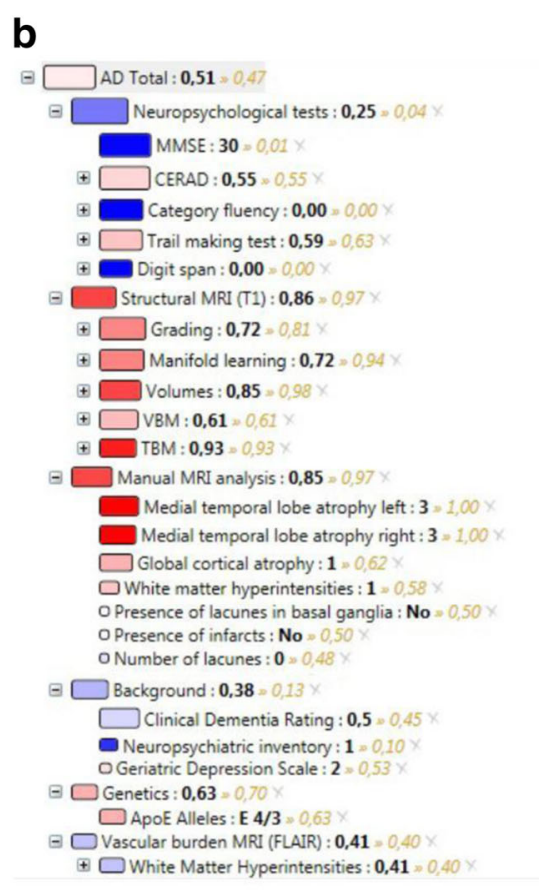

C

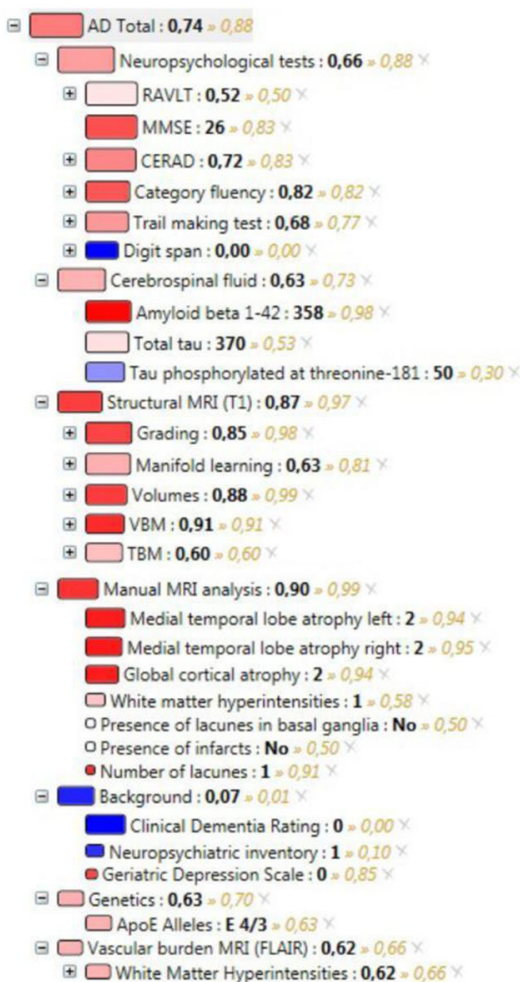

Fig. 2 Disease state fingerprints from the PredictND tool. a A 46-year-old male with a family history of early-onset dementia and 1 year of wordfinding problems, loss of initiative, and subjective complaints of affected memory and sleep. Aß42, $1059 \mathrm{ng} / \mathrm{L}$; total tau, $201 \mathrm{ng} / \mathrm{L}$; and P-tau, 43 $\mathrm{ng} / \mathrm{L}$. Diagnosed with SCD at baseline. The DSI value (0.06) predicted a stable condition and increased the clinician's confidence from a VAS score of $55 \%$ to $80 \%$ in the prediction of stable SCD. After the 18-month follow-up, the diagnosis was still stable SCD. $\mathbf{b}$ A 76-year-old female with mild forgetfulness for words and names during the last 2 years. Normal daily function. MMSE, 30; CERAD learning, 21/30; and CERAD recall, 1/10. Diagnosed with $\mathrm{MCl}$ at baseline. The DSI value (0.51) did not indicate a clear stable or progressive condition. The clinician without tool predicted the follow-up diagnosis to be stable $\mathrm{MCl}$, whereas the clinician with tool predicted the patient to progress to AD dementia. The diagnosis at 24month follow-up was $\mathrm{MCl}$, but after the end of the project at a 3-year follow-up visit, the patient was diagnosed with AD dementia. c A 74-yearold male with memory problems for events and names, loss of initiative, and orientation. A $342,358 \mathrm{ng} / \mathrm{L}$; total tau, $370 \mathrm{ng} / \mathrm{L}$; and P-tau, $50 \mathrm{ng} / \mathrm{L}$. Diagnosed with amnestic $\mathrm{MCl}$ at baseline. The DSI value (0.78) predicted progression and increased the clinician's confidence in the prediction of progression to dementia by $30 \%$ on the VAS scale. After the 12-month follow-up, the patient had progressed to AD dementia 
indication $(n=145)$, and $A \beta 42$, total tau, and tau phosphorylated at threonine 181 were measured with commercially available ELISA (Innotest, Fujirebio Europe, Ghent, Belgium). MRI scans were acquired on either $1.5 \mathrm{~T}$ or $3 \mathrm{~T}$ scanners, including a T1-weighted gradient echo sequence and a fast fluid-attenuated inversion recovery (FLAIR) sequence. Visual MRI rating was performed using the following: global cortical atrophy (GCA), medial temporal lobe atrophy (MTA), and Fazekas scale for white matter hyperintensities (WMH) [3840]. Additionally, the PredictND tool extracted imaging biomarkers from the MRI scans using the following automatic quantification methods: hippocampus segmentation measuring volume, tensor-based morphometry analyzing changes in the local volume, voxel-based morphometry analyzing changes in the local gray matter concentration, manifold learning finding low-dimensional representation of high-dimensional data, region-of-interest (ROI) grading comparing similarity of intensities within ROIs and vascular burden combining volume of white matter hyperintensities (WMH), volume of cortical infarcts, and volume of lacunar infarcts. All methods are described in [34].

\section{Statistical analysis}

Differences in baseline characteristics between groups were assessed using independent $t$ test and chi-square test, where appropriate.

First, we assessed the effect of the PredictND tool on the clinicians' prediction of progression. Baseline predictions were defined as either concordant or discordant according to whether the prediction changed or remained unchanged after using the PredictND tool. Moreover, the predictions were defined as correct or incorrect based on whether or not they corresponded to the clinical progression outcome at follow-up. The impact of the PredictND tool on the prognostic accuracy was calculated based on the difference between paired proportions and tested using McNemar's test. Subsequently, we repeated the analysis including only progression to $\mathrm{MCI}$ or dementia due to $\mathrm{AD}$ (excluding other types of dementias). Further, we restricted the analysis to a subset of the cohort with more certain DSI classifications; either high probability of progression (DSI $\geq 0.8$ ) or low probability of progression $(\mathrm{DSI} \leq 0.2)$. For evaluation of performance, we used the following metrics: sensitivity, specificity, negative predictive value (NPV), positive predictive value (PPV), accuracy, and balanced accuracy (defined as the average of sensitivity and specificity). In the DSI classification, the cutoff value for progression was defined as a DSI $\geq 0.5$.

Finally, paired-sample $t$ tests were used to assess change in the level of confidence in the prediction (VAS $0-100 \%)$ after applying the PredictND tool to the evaluation. The level of significance was set at $p$ value $<0.05$ (two-tailed). SAS enterprise guide 7.1 was used for the analyses (SAS Institute, Inc., Cary, NC, USA).

\section{Results \\ Participants}

Baseline characteristics are presented in Table 1. After a mean of $1.7 \pm 0.4$ years, 21 (9\%) of the patients with SCD had progressed to either MCI $(n=16), \mathrm{AD}(n=3)$, or non-AD dementia ( $n=2$, mixed dementia). Of the patients with MCI, 63 (32\%) progressed to AD dementia $(n=41)$ or non-AD dementia $(n=22,4$ frontotemporal dementia, 3 dementia with Lewy bodies, 4 vascular dementia, 5 mixed dementia, 6 other types of dementia). Baseline characteristics according to the outcome at follow-up are presented in Table 2 (stratified by SCD and MCI in Additional file 1: Table S1).

\section{Prediction of progression without and with the PredictND tool}

In $56(13 \%)$ patients, the clinician changed the prediction of progression when using the tool. The prediction changed correctly in $29(7 \%)$ patients and incorrectly in $27(6 \%)$ patients compared to the follow-up diagnosis. The prediction remained unchanged in 373 patients, with $301(70 \%)$ correct and $72(17 \%)$ incorrect predictions (Table 3). Figure 3 shows correctly and incorrectly changed predictions in relation to the DSI values. Lower DSI values were associated with a higher number of patients with correctly changed predictions, whereas higher DSI values were associated with more incorrectly changed predictions.

Table 4 presents the performance of clinicians without and with the PredictND tool, and the DSI classification alone. Across all patients, we found practically no difference in the prognostic accuracy between the clinician without (acc. $=76 \%$ ) and with tool (acc. $=77 \%)(0.4 \%$, $95 \% \mathrm{CI}-3.0 \%$; $+3.9 \%, p=0.79)$. When excluding patients who progressed to non-AD dementia, the difference in prognostic accuracies seemed to increase slightly

Table 1 Baseline characteristics

\begin{tabular}{llll}
\hline Characteristic & SCD & MCl & $p$ value \\
& $n=230$ & $n=199$ & \\
\hline Female, $n(\%)$ & $150(65)$ & $80(40)$ & $<.0001$ \\
Age, years & $64(9)$ & $70(9)$ & $<.0001$ \\
Duration of symptoms, years & $3(4)$ & $2(3)$ & 0.009 \\
MMSE & $29(1)$ & $27(3)$ & $<.0001$ \\
Follow-up time, years & $1.9(0.3)$ & $1.6(0.5)$ & $<.0001$ \\
Progressed, $n(\%)$ & $21(9)$ & $63(32)$ & $<.0001$ \\
Outcome: MCl/AD/non-AD, $n$ & $16 / 3 / 2$ & $-/ 41 / 22$ & $\mathrm{NA}$
\end{tabular}

Differences between groups were assessed using independent $t$ test and chisquare test. Data are presented as mean \pm SD or number (\%) Abbreviations: $S C D$ subjective cognitive decline, $\mathrm{MCl}$ mild cognitive impairment, MMSE Mini-Mental State Examination 
Table 2 Baseline characteristics according to the outcome at follow-up

\begin{tabular}{|c|c|c|c|c|}
\hline \multirow[t]{2}{*}{ Characteristic } & \multirow[t]{2}{*}{$n$} & \multirow{2}{*}{$\begin{array}{l}\text { Stable } \\
n=345\end{array}$} & \multirow{2}{*}{$\begin{array}{l}\text { Progressed } \\
n=84\end{array}$} & \multirow{2}{*}{$\begin{array}{l}p \\
\text { value }\end{array}$} \\
\hline & & & & \\
\hline \multicolumn{5}{|l|}{ Demographics } \\
\hline Female, $n(\%)$ & 429 & $191(55)$ & $39(46)$ & $<.0001$ \\
\hline Age, years & 429 & $65 \pm 9$ & $72 \pm 8$ & $<.0001$ \\
\hline Duration of symptoms, years & 380 & $3 \pm 3$ & $3 \pm 3$ & 0.36 \\
\hline $\mathrm{MCl} / \mathrm{AD} / \mathrm{non}-\mathrm{AD}, n$ & & - & $16 / 44 / 24$ & NA \\
\hline CDR, $n(0.0 / 0.5 / 1.0)$ & 424 & 198/136/6 & $11 / 52 / 4$ & NA \\
\hline \multicolumn{5}{|l|}{ APOE status } \\
\hline APOE e4 carrier, $n(\%)$ & 146 & $50(14)$ & $14(17)$ & 0.12 \\
\hline \multicolumn{5}{|l|}{ Cognitive tests } \\
\hline MMSE & 427 & $28 \pm 2$ & $26 \pm 3$ & $<.0001$ \\
\hline Memory_learning & 420 & $42 \pm 11$ & $31 \pm 10$ & $<.0001$ \\
\hline Memory_recall & 420 & $9 \pm 4$ & $4 \pm 3$ & $<.0001$ \\
\hline TMT-A, seconds & 422 & $42 \pm 19$ & $58 \pm 30$ & $<.0001$ \\
\hline TMT-B, seconds & 402 & $102 \pm 60$ & $164 \pm 82$ & $<.0001$ \\
\hline Fluency_animal & 407 & $23 \pm 7$ & $18 \pm 6$ & $<.0001$ \\
\hline Fluency_letter & 377 & $14 \pm 5$ & $12 \pm 5$ & 0.006 \\
\hline Clock-drawing & 394 & $3 \pm 1$ & $2 \pm 1$ & $<.0001$ \\
\hline \multicolumn{5}{|l|}{ CSF } \\
\hline $\mathrm{A} \beta 42, \mathrm{pg} / \mathrm{ml}$ & 145 & $933 \pm 285$ & $748 \pm 338$ & 0.002 \\
\hline P-tau, pg/ml & 145 & $53 \pm 24$ & $63 \pm 33$ & 0.05 \\
\hline Total tau, pg/ml & 145 & $348 \pm 197$ & $445 \pm 318$ & 0.03 \\
\hline \multicolumn{5}{|l|}{ MRI—visual scores } \\
\hline GCA (median, Q1-Q3) & 418 & $0.7 \pm 0.7(1,0-1)$ & $1.2 \pm 0.8(1,1-2)$ & $<.0001$ \\
\hline MTA, right (median, Q1-Q3) & 398 & $0.6 \pm 0.8(0,0-1)$ & $1.4 \pm 1.0(1,1-2)$ & $<.0001$ \\
\hline MTA, left (median, Q1-Q3) & 398 & $0.6 \pm 0.8(0,0-1)$ & $1.6 \pm 1.0(1,1-2)$ & $<.0001$ \\
\hline Fazekas score (median, Q1-Q3) & 420 & $0.8 \pm 0.8(1,0-1)$ & $1.1 \pm 0.8(1,1-2)$ & 0.009 \\
\hline
\end{tabular}

Differences between groups were assessed using independent $t$ test and chi-square test. Data are presented as mean \pm SD unless otherwise specified Abbreviations: CDR clinical dementia rating (global score, range 0-3); MMSE Mini-Mental State Examination; Memory Rey Auditory Verbal Learning Test (RAVLT) values, using $z$-scoring for those with only the Consortium to Establish a Registry for Alzheimer's Disease (CERAD) word list memory test; TMT Trail Making Test; CSF cerebrospinal fluid; $A \beta 42$ amyloid beta 1-42; P-tau tau phosphorylated at threonine 181; MRI magnetic resonance imaging; GCA global cortical atrophy; MTA medial temporal lobe atrophy

$(1.2 \%, 95 \% \mathrm{CI}-2.2 \% ;+4.7 \% ; p=0.48)$. Including only patients with DSI below 0.2 and above $0.8(n=203)$, the accuracy increased by $3.0 \%(95 \% \mathrm{CI}-0.6 \%$; $+6.5 \%, p=$ 0.11 ) from 90 to $93 \%$ (Table 4). Overall, the PPVs were moderate, whereas the NPVs were high. A slightly higher NPV (0.96) and lower PPV (0.34 and 0.35) were observed for SCD compared to MCI (NPV $=0.78$ and 0.81 , and PPV $=0.48$ ).

The DSI classification alone performed at the same level as the clinicians. For the subgroups with DSI values below 0.2 or above 0.8 , representing a more certain DSI classification for either progression or stability, the DSI classification alone had significant higher accuracy than the clinicians without tool $(6.4 \%, 95 \%$ CI $2.1 \% ; 10.7 \% ; p$ $=0.004$ ) (additional results in Additional file 1: Tables $\mathrm{S} 2-\mathrm{S} 7)$.

\section{Confidence in the prediction of progression without and} with the PredictND tool

In $34 \%(n=144)$ of all patients, the level of confidence in the prediction of progression changed following the application of the PredictND tool. The confidence increased from low to moderate/high or from moderate to high in $19 \%(n=83)$ of the correct and $5 \%(n=23)$ of the incorrect predictions meaning that $78 \%$ of the 106 cases with increased confidence supported a correct prediction. The confidence decreased from high to moderate/low or from moderate to low in $6 \%(n=25)$ of the correct and $3 \%(n=13)$ of the incorrect predictions (Table 3).

Overall, confidence in the prediction increased significantly with $4 \%$ on the VAS scale $(p<.0001)$ when applying the PredictND tool to the evaluation. For patients 
Table 3 Impact of the PredictND tool on the baseline prediction of progression

\begin{tabular}{|c|c|c|c|c|c|c|}
\hline \multirow{2}{*}{$\frac{\text { With tool prediction (WT) }}{\text { According to FU diagnosis, }}$} & \multicolumn{2}{|c|}{ All $(n=429)$} & \multicolumn{2}{|c|}{$\operatorname{SCD}(n=230)$} & \multicolumn{2}{|c|}{$\mathrm{MCl}(n=199)$} \\
\hline & Correct & Incorrect & Correct & Incorrect & Correct & Incorrect \\
\hline$n(\%)$ & $330(77)$ & $99(23)$ & $197(86)$ & $33(14)$ & $133(67)$ & $66(33)$ \\
\hline Unchanged prediction, $\mathrm{WOT}=\mathrm{WO}, \mathrm{n}(\%)$ & $301(70)$ & $72(17)$ & $184(80)$ & $21(9)$ & $117(59)$ & $51(26)$ \\
\hline Changed prediction, WOT $\neq$ WO, $n(\%)$ & $29(7)$ & $27(6)$ & $13(6)$ & $12(5)$ & $16(8)$ & $15(7)$ \\
\hline \multicolumn{7}{|l|}{ Confidence in VAS score (0-100\%) } \\
\hline Without tool confidence (WOT) & $67 \pm 15$ & $60 \pm 15$ & $72 \pm 15$ & $61 \pm 18$ & $60 \pm 12$ & $60 \pm 13$ \\
\hline With tool confidence (WT) & $72 \pm 16$ & $62 \pm 17$ & $79 \pm 13$ & $63 \pm 19$ & $62 \pm 14$ & $61 \pm 15$ \\
\hline$\Delta$ Difference confidence & $5 \pm 13^{*}$ & $2 \pm 15$ & $7 \pm 10^{*}$ & $2 \pm 17$ & $2 \pm 16$ & $1 \pm 14$ \\
\hline \multicolumn{7}{|l|}{ Confidence (high/moderate/low) } \\
\hline Increase in confidence (\%) & $83(19)$ & $23(5)$ & $54(23)$ & $9(4)$ & $29(15)$ & $14(7)$ \\
\hline Decrease in confidence (\%) & $25(6)$ & $13(3)$ & $8(3)$ & $6(3)$ & $17(9)$ & $7(3)$ \\
\hline Stable confidence (\%) & $222(52)$ & $63(15)$ & $135(59)$ & $18(8)$ & $87(44)$ & $45(22)$ \\
\hline
\end{tabular}

The baseline predicted follow-up diagnosis with tool compared to the follow-up diagnosis for all patients and stratified according to baseline SCD and MCI diagnosis. "Unchanged prediction" indicates patients where the prediction did not change after the PredictND tool was used, whereas in "changed prediction," the baseline predicted follow-up diagnosis without tool was changed when applying the tool. Data are presented as mean \pm SD or $n$ (\%). Difference between without and with tool confidence was assessed using paired-sample $t$ tests

Abbreviations: WOT without tool, WT with tool, $\Delta$ difference confidence the difference between confidence in the prediction without and with tool, VAS visual analogue scale from 0 to $100 \%$

*Significant increased confidence after using the PredictND tool, $p<0.05$

with correct predictions, the mean VAS score increased $5 \%(p<.0001)$ and for incorrect predictions the score increased by $2 \%(p=0.33)$ (Table 3$)$. The increase in the mean VAS score was highest for SCD patients with correct predictions $(\triangle \mathrm{VAS}=7 \%, p<.0001)$. Furthermore, in patients with more extreme DSI values (DSI $<0.2$ or DSI $>0.8$ ), the clinician's confidence in the predictions increased more $(\triangle \mathrm{VAS}=7 \%, p<.0001)$ than in patients with medium DSI values $(\Delta \mathrm{VAS}=-2 \%, p=0.07)(p$ $<.0001)$.

\section{Discussion}

In this prospective multicenter study, we found that the prediction of progression in non-demented memory

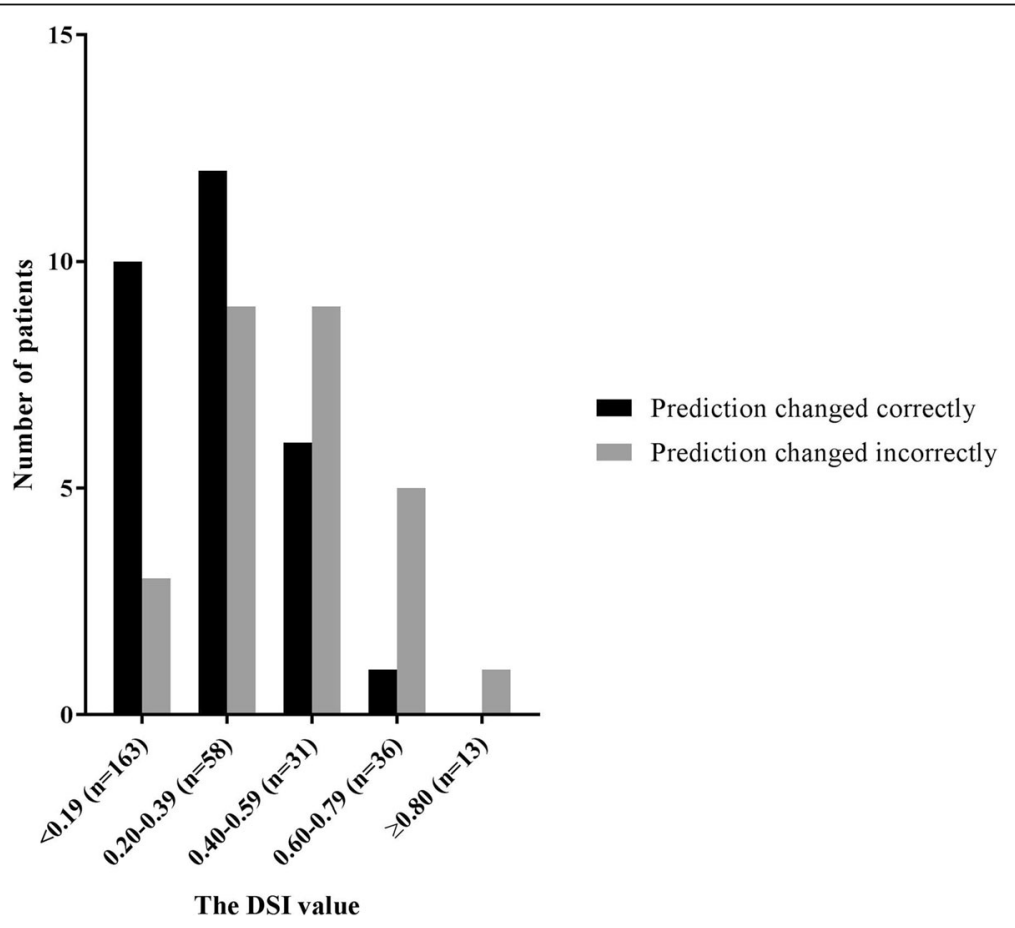

Fig. 3 The number of changed predictions after application of the PredictND tool, stratified by DSI values. DSI disease state index 
Table 4 Performance to predict progression for clinicians without and with the PredictND tool, and the DSI classification alone

\begin{tabular}{|c|c|c|c|c|c|c|}
\hline Cohort & SN & SP & PPV & NPV & Accuracy & Bal. Acc. \\
\hline \multicolumn{7}{|l|}{ All $(n=429)$} \\
\hline Without tool & 0.67 & 0.79 & 0.43 & 0.91 & 0.76 & 0.73 \\
\hline With tool & 0.60 & 0.81 & 0.43 & 0.89 & 0.77 & 0.70 \\
\hline DSI & 0.63 & 0.83 & 0.47 & 0.90 & 0.79 & 0.73 \\
\hline \multicolumn{7}{|c|}{ Excl. non-AD dementia $(n=405)$} \\
\hline Without tool & 0.67 & 0.79 & 0.35 & 0.93 & 0.77 & 0.73 \\
\hline With tool & 0.62 & 0.81 & 0.36 & 0.92 & 0.78 & 0.71 \\
\hline DSI & 0.63 & 0.83 & 0.39 & 0.93 & 0.80 & 0.73 \\
\hline \multicolumn{7}{|l|}{$\operatorname{SCD}(n=230)$} \\
\hline Without tool & 0.67 & 0.87 & 0.34 & 0.96 & 0.85 & 0.77 \\
\hline With tool & 0.67 & 0.88 & 0.35 & 0.96 & 0.86 & 0.77 \\
\hline DSI* & 0.33 & 0.96 & 0.47 & 0.93 & 0.90 & 0.65 \\
\hline \multicolumn{7}{|l|}{$\mathrm{MCl}(n=199)$} \\
\hline Without tool & 0.67 & 0.66 & 0.48 & 0.81 & 0.66 & 0.66 \\
\hline With tool & 0.57 & 0.71 & 0.48 & 0.78 & 0.67 & 0.64 \\
\hline DSI & 0.73 & 0.62 & 0.47 & 0.83 & 0.65 & 0.67 \\
\hline \multicolumn{7}{|c|}{$\mathrm{DSI} \leq 0.2$ or $\mathrm{DSI} \geq 0.8(n=203)$} \\
\hline Without tool & 0.67 & 0.92 & 0.44 & 0.97 & 0.90 & 0.79 \\
\hline With tool & 0.61 & 0.96 & 0.58 & 0.96 & 0.93 & 0.78 \\
\hline DSI & 0.78 & 0.98 & 0.78 & 0.98 & $0.96^{*}$ & 0.88 \\
\hline
\end{tabular}

Abbreviations: Prog conversion of SCD to $\mathrm{MCl}, \mathrm{AD}$ or another type of dementia, and $M C l$ to $A D$ or another type of dementia; $S N$ sensitivity; $S P$ specificity; $P P V$ positive predictive value; NPV negative predictive value; Bal.Acc. balanced accuracy; DSI disease state index; $S C D$ subjective cognitive decline; $M C I$ mild cognitive impairment

**Significant difference between without tool and DSI classification accuracy, $p=0.012$

*Results from different cutoff values are available in Additional file 1: Table S8

clinic patients was changed in $13 \%$ of all patients when the PredictND tool was applied. Particularly for patients with extreme DSI values (indicating either progression or stability with higher certainty), the use of the tool had a tendency to increase the prognostic accuracy and the DSI alone showed statistically significant improvement. Moreover, the clinicians' confidence in the prediction increased when the PredictND tool was added to the evaluation, especially for patients with SCD.

In previous studies using retrospective data, we have shown that the DSI classifier can discriminate between stable and progressive MCI (acc. $=0.70-0.71$ ), as well as SCD (balanced acc. $=0.74)[21-23,25]$. Moreover, DSI was able to identify half of the patients with MCI who progressed to a clinical $\mathrm{AD}$ diagnosis 12 months prior to the conversion with an accuracy of $88 \%$ [20]. The accuracy for MCI (acc. $=0.66-0.67)$ in the current multicenter study was similar to previous results in the retrospective studies, e.g., $0.65-0.75$ in [37]. Furthermore, in this study, we extended the previous findings by applying the
PredictND tool to a prospective mixed memory clinic cohort to evaluate the real-life impact of the tool.

Focusing on the patients with a higher certainty in the classification (DSI $<0.2$ or $>0.8$ ), which accounted for nearly half of the cohort, we found that use of the PredictND tool had a tendency to improve the prognostic accuracy $(p=0.11)$. However, when the tool was used alone in this patient group, a statistically significant difference was observed. This implies that clinicians could have trusted the tool more than they did when assessing the cases with extreme DSI values. The patients with DSI between 0.2 and 0.8 form an inconclusive group for which accurate prediction is not possible with given data. In previous studies, we have likewise found higher performance with more extreme DSI values, especially lower values (DSI <0.2), emphasizing a clear strength of the tool for identifying patients who will remain stable $[20,21,23,25]$. Our results showed high NPV, whereas the PPV was relatively low both for clinicians without and with tool, and for the DSI value alone [18, 25]. Therefore, when evaluating the whole cohort, the major value of the tool seems to be in establishing reassurance for patients who are unlikely to progress. However, for the extreme DSI values, the PPV was higher when the clinician was assisted by the tool mainly due to less false positive predictions. For this subgroup, the tool thus seems more precise in identifying the individuals at high risk of progression (risk ratios 5.0-8.8, see Additional file 1: Table S4) with a need for a closer follow-up within the clinically relevant period of 12-24 months.

High confidence in the prognosis is important to provide convincing reassurance to patients with a stable condition and to identify patients in need of comprehensive clinical follow-up or even early treatment. We found that the clinicians' confidence in their prediction increased when using the PredictND tool. The highest increase in confidence was seen in correct predictions, especially for SCD patients. Moreover, as expected, the change in confidence was dependent on the DSI value with higher impact of the tool when the classification was more consistent.

The main strength of the study is the large well-characterized multicenter cohort making the result more generalizable. Moreover, the prospective design provided optimal conditions to test the tool in an actual clinical setting when a clinician has seen or obtained second-hand information of the patient.

Prospective studies are also associated with several potential limitations. First, the study design was a trade-off between the importance of retaining the clinician's impression of the patient and minimizing bias carried over from the first to the second evaluation. Thus, in some cases, the time between the evaluations was longer than intended and it might have affected the result. 
Furthermore, to evaluate progression in patients with MCI and especially SCD, the follow-up time was short $[1,5,18]$. Though, for clinical use particularly in patients with $\mathrm{MCI}$, it may be more relevant to identify the impact on prediction of progression within 1-2 years rather than distant future. Generally, the number of progressors was low, but it corresponded to estimated annual conversion rates in other studies $[1,5]$. In a few patients, the clinical condition improved, and these cases were handled as stable in the analysis as the tool's impact in terms of reassurance was considered similar. Relatively broad entry criteria may have also led to inclusion of patients with mild dementia rather than strictly MCI. However, baseline characteristics (e.g., MMSE and CDR) indicate that this was seldomly the case. Another issue is the use of training data from controls and AD patients. However, our previous studies demonstrate that the classification performance is comparable independent on whether the model is trained with data from SCD and AD patients or from stable and progressive MCI patients $[19,20,25]$. Yet, when the clinician interprets the DSI values, the choice of $\mathrm{AD}$ and controls as training data might be associated with some considerations. For MCI patients, the DSI values are closer to the cutoff value $(\mathrm{DSI}=0.5)$ than if the model was trained using stable and progressive MCI subjects [19, 25]. For SCD patients considering progression to $\mathrm{MCI}$, the cutoff value (DSI = 0.5 ) is not optimal as many MCI patients will have a DSI value lower than 0.5 . This explains why sensitivity was low (0.33) and specificity was high (0.96) in the SCD group using DSI (performance at different cutoff values for SCD is presented in Additional file 1: Table S8). In this study, the cutoff was not optimized separately for SCD and MCI patients but the same cutoff (DSI $=0.5$ ) was used for all patients. Refinement of the prediction algorithm accounting for, e.g., different cutoff values may improve the results. Finally, specific models for non-AD dementias were not applied and our analysis excluding patients who progressed to non-AD dementia showed a slight increase in the prognostic accuracy (see Additional file 1: Table S2), suggesting that training data with cohorts of non-AD dementias may improve the clinical importance of the tool.

Various studies with cognitive tests, CSF, and MRI biomarkers have shown the prognostic value of diagnostic tests on group levels, whereas predictive classifiers have the advantage of providing predictions on an individual patient level with MCI and SCD [2, 3, 9-13]. In additional, the PredictND tool, based on the DSI classifier, also analyzes large quantities of heterogeneous patient data and enables the clinician to easily interpret the results visually $[19,26]$. Other commonly used classifiers are logistic regression models, Bayesian classifiers, support vector machine (SVM), and random forest [19, 41-
44]. Another promising prognostic model interpreting MRI and CSF in the light of age, gender, and MMSE to provide a progression risk has recently been introduced for clinical use, but to our knowledge, the clinical impact has not yet been investigated [18]. These and other emerging prognostic models represent potentially very valuable clinical support tools for the clinicians in the future. However, to ensure optimal quality for the patients, it is important to evaluate and compare the performance and clinical impact, and preferably in a standardized manner [17].

\section{Conclusions}

Findings from this study indicate that the PredictND tool affected the prediction of progression for SCD and MCI both in terms of changing the clinicians' predictions and increasing their confidence. Although no statistically significant difference was observed when using the tool, the results show potential for improvements especially for patients with most extreme DSI values (DSI classifications $<0.2$ or $>0.8$ ). The tool alone showed an increase in accuracy (statistically significant) compared with the situation when no tool was used in the patients with DSI $<0.2$ or DSI $>0.8$. In this subpopulation, stable patients were identified with high accuracy. Furthermore, our results indicate that decision support tools in the future could make clinicians more confident in their short-term prognosis by providing a decent second opinion in prognostic decision-making.

\section{Additional files}

Additional file 1: Presents additional results, such as demographics, accuracy, and performance for subgroups. (PDF $2187 \mathrm{~kb}$ )

\section{Abbreviations}

18F-FDG-PET: 18F-fluorodeoxyglucose positron emissions tomography; AD: Alzheimer's disease; A 42 : Amyloid- $\beta$ 1-42; CDR: Clinical dementia rating; CSF: Cerebrospinal fluid; DSF: Disease state fingerprint; DSI: Disease state index; FLAIR: Fluid-attenuated inversion recovery; MCl: Mild cognitive impairment; MRI: Magnetic resonance imaging; MTA: Medial temporal lobe atrophy; NPV: Negative predictive value; PPV: Positive predictive value; ROI: Region-of-interest; SCD: Subjective cognitive decline; VAS: Visual analogue scale; WMH: White matter hyperintensities

\section{Acknowledgements}

The PredictND consortium consisted of collaborates from VTT Technical Research Centre of Finland, GE Healthcare Ltd, Imperial College London, Alzheimer Europe, Alzheimer Center - VU University Medical Center, Amsterdam, the Netherlands, the Danish Dementia Research Centre, Copenhagen University Hospital, Denmark, the department of Gerontology and Geriatrics of the University of Perugia, 'S. Maria della Misericordia' Hospital of Perugia, Italy, the department of Neurology from the University of Eastern Finland.

\section{Funding}

This work was co-funded by the European Commission under grant agreement 611005 (PredictND). For development of the PredictND tool, VTT Technical Research Center of Finland Ltd has received funding from European Union's Seventh Framework Programme for research, technological 
development and demonstration under grant agreements 601055 (VPH-DARE@IT), 224328 and 611005 . Furthermore, the work was supported by the Ellen Mørch's foundation and the family Hede Nielsen's foundation.

\section{Availability of data and materials}

Data are available from the authors upon reasonable request.

\section{Authors' contributions}

$\mathrm{MBr}$ has contributed to the study concept and design, drafted the manuscript, and interpreted data. KSF has contributed to the study concept and design, revised the manuscript for intellectual content, and supervised the project. HFMR, MBa, LG, ND, BBA, AWL, MH, SK, SH, AMR, GW, PM, HS, and WMvdF have contributed to the collection of the data and have revised the manuscript for intellectual content. JK, TU, AT, MvG, and DR have contributed to the development of the PredictND tool and revised the manuscript for intellectual content. $J$ has contributed to the development of the PredictND tool, interpreted data, and revised the manuscript for intellectual content. SGH has contributed to the study concept and design. All authors read and approved the final manuscript.

\section{Ethics approval and consent to participate}

All patients provided written informed consent for their data to be used for research purposes. The project was approved by the local Medical Ethical Committee in all four European clinical centers: the Regional Committees on Medical Research Ethics of the Capital Region of Denmark (Approval no.: $\mathrm{H}$ 1-2014-126), CEAS-Umbria (Comitato Etico Aziende Sanitarie-Umbria), Italy (Approval no.: CEAS 2381/14), the Ethical Committee of the VUmc, Amsterdam, the Netherland (Approval no: 2014-12 amendment to protocol 2015.16, at 18-12-2014), and the Ethical Committee of Northern Savo Hospital District, Kuopio, Finland (Approval no: 71/2014, 29.10.2014). Furthermore, the protocol was approved by the Data Protection Agency in Finland and subsequently in Denmark (Journal no.: 2012-58-0004).

\section{Consent for publication}

Not applicable.

\section{Competing interests}

HS has served in advisory boards for ACImmune and MERK. WMvdF performs contract research for Biogen and has research programs funded by the ZonMW, NWO, EU-FP7, Alzheimer Nederland, CardioVascular Onderzoek Nederland, Stichting Dioraphte, Gieskes-Strijbis Fonds, Boehringer Ingelheim, Piramal Neuroimaging, Roche BV, Janssen Stellar, and Combinostics. All funding is paid to her institution. $J \mathrm{~L}$ and $J K$ are shareholders in Combinostics Oy that owns the following IPR related to the patent: (1) J. Koikkalainen and J. Lotjonen. A method for inferring the state of a system, US7,840,510 B2, PCT/ FI2007/050277. (2) J. Lotjonen, J. Koikkalainen and J. Mattila. State Inference in a heterogeneous system, PCT/FI2010/050545. FI20125177. The other authors declare that they have no competing interests.

\section{Publisher's Note}

Springer Nature remains neutral with regard to jurisdictional claims in published maps and institutional affiliations.

\section{Author details}

'Danish Dementia Research Centre, Neuroscience Centre, Department of Neurology, Rigshospitalet, University of Copenhagen, Copenhagen University Hospital, Blegdamsvej 9, 2100 Copenhagen, Denmark. ${ }^{2}$ Alzheimer Center Amsterdam, Department of Neurology, Amsterdam Neuroscience, Vrije Universiteit Amsterdam, Amsterdam UMC, Amsterdam, The Netherlands. ${ }^{3}$ Institute of Gerontology and Geriatrics, University of Perugia, Perugia, Italy. ${ }^{4}$ Combinostics Ltd., Tampere, Finland. ${ }^{5} \mathrm{VTT}$ Technical Research Centre of Finland Ltd, Tampere, Finland. ${ }^{6}$ Department of Computing, Imperial College London, London, UK. ${ }^{7}$ Neurology, Institute of Clinical Medicine, University of Eastern Finland, Kuopio, Finland. ${ }^{8}$ Medical Research Center, Oulu University Hospital, Oulu, Finland. ${ }^{9}$ Neurology, Neuro Center, Kuopio University Hospital Kuopio, Finland. ${ }^{10}$ Neurology, Unit of Clinical Neuroscience, University of Oulu, Oulu, Finland.
Received: 21 November 2018 Accepted: 11 March 2019

Published online: 20 March 2019

\section{References}

1. Mitchell AJ, Beaumont H, Ferguson D, et al. Risk of dementia and mild cognitive impairment in older people with subjective memory complaints: meta-analysis. Acta Psychiatr Scand. 2014;130:439-51.

2. Visser PJ, Verhey F, Knol DL, et al. Prevalence and prognostic value of CSF markers of Alzheimer's disease pathology in patients with subjective cognitive impairment or mild cognitive impairment in the DESCRIPA study: a prospective cohort study. Lancet Neurol. 2009;8:619-27 https://doi.org/10. 1016/S1474-4422(09)70139-5

3. Jessen $F$, Amariglio RE, Van Boxtel $M$, et al. A conceptual framework for research on subjective cognitive decline in preclinical Alzheimer's disease. Alzheimers Dement. 2014;10:844-52. https://doi.org/10.1016/j.jalz.2014.01. 001.

4. Albert MS, DeKosky ST, Dickson D, et al. The diagnosis of mild cognitive impairment due to Alzheimer's disease: recommendations from the National Institute on Aging-Alzheimer's Association workgroups on diagnostic guidelines for Alzheimer's disease. Alzheimers Dement. 2011;7: 270-9.

5. Mitchell AJ, Shiri-Feshki M. Rate of progression of mild cognitive impairment to dementia - meta-analysis of 41 robust inception cohort studies. Acta Psychiatr Scand. 2009:119:252-65.

6. Frank L, Lloyd A, Flynn JA, et al. Impact of cognitive impairment on mild dementia patients and mild cognitive impairment patients and their informants. Int Psychogeriatrics. 2006;18:151-62.

7. Kunneman M, Smets EMA, Bouwman FH, et al. Clinicians' views on conversations and shared decision making in diagnostic testing for Alzheimer's disease: the ABIDE project. Alzheimers Dement Transl Res Clin Interv. 2017:3:305-13.

8. Jack CR, Knopman DS, Jagust WJ, et al. Tracking pathophysiological processes in Alzheimer's disease: an updated hypothetical model of dynamic biomarkers. Lancet Neurol. 2013;12:207-16. https://doi.org/10.1016/ S1474-4422(12)70291-0.

9. Vos SJB, Van Rossum IA, Verhey F, et al. Prediction of Alzheimer disease in subjects with amnestic and nonamnestic MCl. Neurology. 2013;80:1124-32. https://doi.org/10.1212/WNL.0b013e318288690c

10. Herukka S-K, Hviid Simonsen A, Andreasen N et al. Perspective recommendations for cerebrospinal fluid Alzheimer's disease biomarkers in the diagnostic evaluation of mild cognitive impairment. Alzheimers Dement. 2017;13:285-95. https://doi.org/10.1016/j.jalz.2016.09.009.

11. Van Harten AC, Visser PJ, Pijnenburg YAL, et al. Cerebrospinal fluid Aß42 is the best predictor of clinical progression in patients with subjective complaints. Alzheimers Dement. 2013;9:481-7. https://doi.org/10.1016/j.jalz. 2012.08.004

12. Davatzikos $C$, Bhatt $P$, Shaw $L M$, et al. Prediction of $M C I$ to $A D$ conversion, via MRI, CSF biomarkers, and pattern classification. Neurobiol Aging. 2011; 32:2322.e19-27. https://doi.org/10.1016/j.neurobiolaging.2010.05.023.

13. Madureira S, Verdelho A, Moleiro C, et al. Neuropsychological predictors of dementia in a three-year follow-up period: data from the LADIS study on behalf of the LADIS study. Dement Geriatr Cogn Disord. 2010;29:325-34 https://doi.org/10.1159/000278333.

14. Alexopoulos P, Werle L, Roesler J, et al. Conflicting cerebrospinal fluid biomarkers and progression to dementia due to Alzheimer's disease. Alzheimers Res Ther. 2016:8:1-10. https://doi.org/10.1186/s13195-016-0220-z.

15. van der Flier WM, Kunneman M, Bouwman FH, et al. Diagnostic dilemmas in Alzheimer's disease: room for shared decision making. Alzheimers Dement Transl Res Clin Interv. 2017;3:301-4. https://doi.org/10.1016/j.trci. 2017.03.008.

16. Oxtoby NP, Alexander DC. Imaging plus X: multimodal models of neurodegenerative disease. Curr Opin Neurol. 2017;30:371-9. https://doi.org/ 10.1097/WCO.0000000000000460.

17. Bron EE, Smits M, Van Der Flier WM, et al. Standardized evaluation of algorithms for computer-aided diagnosis of dementia based on structural MRI: the CADDementia challenge, for the Alzheimer's Disease Neuroimaging Initiative 1. Neuroimage. 2015;111:562-79. https://doi.org/10. 1016/j.neuroimage.2015.01.048

18. Van Maurik IS, Zwan MD, Tijms BM, et al. Interpreting biomarker results in individual patients with mild cognitive impairment in the Alzheimer's 
Biomarkers in Daily Practice (ABIDE) project. JAMA Neurol. 2017;74:1481-91. https://doi.org/10.1001/jamaneurol.2017.2712.

19. Mattila J, Koikkalainen J, Virkki A, et al. A disease state fingerprint for evaluation of Alzheimer's disease. J Alzheimers Dis. 2014;27:163-76. https:// doi.org/10.3233/JAD-2011-110365.

20. Mattila J, Soininen H, Koikkalainen J, et al. Optimizing the diagnosis of early Alzheimer's disease in mild cognitive impairment subjects. J Alzheimers Dis. 2012;32:969-79. https://doi.org/10.3233/JAD-2012-120934.

21. Liu Y, Mattila J, Ruiz MÁM, et al. Predicting AD conversion: comparison between prodromal AD guidelines and computer assisted PredictAD tool. PLoS One. 2013;8:e55246: 1-8. https://doi.org/10.1371/journal.pone.0055246.

22. Muñoz-Ruiz MÁ, Hall A, Mattila J, et al. Comparing predictors of conversion to Alzheimer's disease using the disease state index. Neurodegener Dis. 2014;13:200-2. https://doi.org/10.1159/000354074.

23. Hall A, Mattila J, Koikkalainen J, Lötjonen J, Wolz R, Scheltens P, Frisoni G, Tsolaki M, Nobili F, Freund-Levi Y, Minthon L, Frölich L, Hampel H, Visser PJS. Predicting progression from cognitive impairment to Alzheimer's disease with the Disease State Index. Curr Alzheimer Res. 2015;12:69-79. https://doi. org/10.2174/1567205012666141218123829.

24. Simonsen A, Mattila J, Hejl A, et al. Application of the PredictAD software tool to predict progression in patients with mild cognitive impairment. Dement Geriatr Cogn Disord. 2012;34:344-50. https://doi.org/10.1159/ 000345554.

25. Rhodius-Meester HFM, Liedes H, Koikkalainen J, et al. Computer-assisted prediction of clinical progression in the earliest stages of AD. Alzheimers Dement Diagnosis Assess Dis Monit. 2018:1-11. https://doi.org/10.1016/j. dadm.2018.09.001.

26. Mattila J, Koikkalainen J, Virkki A, et al. Design and application of a generic clinical decision support system for multiscale data. IEEE Trans Biomed Eng 2012;59:234-40. https://doi.org/10.1109/TBME.2011.2170986.

27. Doyle OM, Westman E, Marquand AF, et al. Predicting progression of Alzheimer's disease using ordinal regression. PLoS One. 2014;9:1-10. https:// doi.org/10.1371/journal.pone.0105542.

28. Ewers M, Walsh C, Trojanowski JQ, et al. Prediction of conversion from mild cognitive impairment to Alzheimer's disease dementia based upon biomarkers and neuropsychological test performance. Neurobiol Aging. 2012;33:1203-1214.e2. https://doi.org/10.1016/j.neurobiolaging.2010.10.019.

29. Bruun M, Gjerum L, Frederiksen KS, et al. Data-driven diagnosis of dementia disorders: the predictND validation study. Alzheimers Dement. 2017;13:4057. https://doi.org/10.1016/j.jalz.2017.06.391.

30. McKhann GM, Knopman DS, Chertkow H, et al. The diagnosis of dementia due to Alzheimer's disease: recommendations from the National Institute on Aging-Alzheimer's association workgroups on diagnostic guidelines for Alzheimer's disease. Alzheimers Dement. 2011;7:263-9. https://doi.org/10. 1016/j.jalz.2011.03.005.

31. Neary D, Snowden JS, Gustafson L, et al. Frontotemporal lobar degeneration: a consensus on clinical diagnostic criteria. Neurology. 1998; 51:1546-54. https://doi.org/10.1212/WNL.51.6.1546.

32. Mckeith I, Dickson D, Lowe J, et al. Diagnosis and management of dementia with Lewy bodies: third report of the DLB consortium. Neurology. 2005;65: 1863-72.

33. Román G, Tatemichi T, Erkinjuntti T, et al. Vascular dementia: diagnostic criteria for research studies: report of the NINDS-AIREN International Workshop. Neurology. 1993;43:250-60. https://doi.org/10.1212/WNL.43.2.250.

34. Koikkalainen J, Rhodius-Meester $\mathrm{H}$, Tolonen A, et al. Differential diagnosis of neurodegenerative diseases using structural MRI data. Neurolmage Clin. 2016;11:435-49. https://doi.org/10.1016/..nicl.2016.02.019.

35. Tolonen A, Rhodius-meester HFM, Bruun M, et al. Data-driven differential diagnosis of dementia using multiclass disease state index classifier. Front Aging Neurosci. 2018;10:1-11. https://doi.org/10.3389/fnagi.2018.00111.

36. Rhodius-meester HFM, Koikkalainen J, Mattila J, et al. Integrating biomarkers for underlying Alzheimer's disease in mild cognitive impairment in daily practice: comparison of a clinical decision support system with individual biomarkers. J Alzheimers Dis. 2016;50:261-70. https://doi.org/10.3233/JAD150548.

37. Hall A, Muñoz-Ruiz M, Mattila J, et al. Generalizability of the disease state index prediction model for identifying patients progressing from mild cognitive impairment to Alzheimer's disease. J Alzheimers Dis. 2015;44:7992. https://doi.org/10.3233/JAD-140942.
38. Pasquier F, Leys D, Weerts JGE, et al. Inter-and intraobserver reproducibility of cerebral atrophy assessment on MRI scans with hemispheric infarcts. Eur Neurol. 1996;36:268-72. https://doi.org/10.1159/000117270.

39. Scheltens P, Van De Pol L. Atrophy of medial temporal lobes on MRI in 'probable' Alzheimer's disease and normal ageing: diagnostic value and neuropsychological correlates. J Neurol Neurosurg Psychiatry. 2012;83:103840.

40. Fazekas F, Chawluk JB, Alavi A, et al. Mr signal abnormalities at 1.5-T in Alzheimer's dementia and normal aging. Am J Roentgenol. 1987;149:351-6. https://doi.org/10.2214/ajr.149.2.351.

41. Shaffer JL, Petrella JR, Sheldon FC, et al. Predicting cognitive decline in subjects at risk for Alzheimer disease by using combined cerebrospinal fluid, MR imaging, and PET. Biomarkers. 2013;266. https://doi.org/10.1148/radiol. 12120010/-/DC1.

42. Cui Y, Liu B, Luo S, et al. Identification of conversion from mild cognitive impairment to Alzheimer's disease using multivariate predictors. PLoS One. 2011;6:2-11. https://doi.org/10.1371/journal.pone.0021896.

43. Bron EE, Smits M, Papma JM, et al. Multiparametric computer-aided differential diagnosis of Alzheimer's disease and frontotemporal dementia using structural and advanced MRI. Eur Radiol. 2017;27:3372-82. https://doi. org/10.1007/s00330-016-4691-x.

44. Falahati F, Westman E, Simmons A. Multivariate data analysis and machine learning in Alzheimer's disease with a focus on structural magnetic resonance imaging. J Alzheimers Dis. 2014;41:685-708. https://doi.org/10. 3233/JAD-131928

\section{Ready to submit your research? Choose BMC and benefit from:}

- fast, convenient online submission

- thorough peer review by experienced researchers in your field

- rapid publication on acceptance

- support for research data, including large and complex data types

- gold Open Access which fosters wider collaboration and increased citations

- maximum visibility for your research: over $100 \mathrm{M}$ website views per year

At BMC, research is always in progress.

Learn more biomedcentral.com/submissions 